DRAFT VERSION MARCH 14, 2021

Typeset using LATEX twocolumn style in AASTeX61

\title{
FORMATION OF BLACK HOLE X-RAY BINARIES WITH NON-DEGENERATE DONORS IN GLOBULAR CLUSTERS
}

\author{
Natalia IVAnova, ${ }^{1,2}$ Cassio A. DA Rocha, ${ }^{1,3}$ Kenny X. VAn, ${ }^{1,2}$ And Jose L.A. NAndeZ ${ }^{1,4}$
}

\author{
${ }^{1}$ Department of Physics, University of Alberta, Edmonton, AB, T6G 2E7, Canada \\ ${ }^{2}$ Kavli Institute for Theoretical Physics, UCSB, Santa Barbara, CA 93106, USA \\ ${ }^{3}$ Instituto de Física, Universidade Federal de Goiś, Goiânia, Goiás, 74.690-900, Brazil \\ ${ }^{4}$ SHARCNET, Faculty of Science, University of Western Ontario, London, ON, N6A 5B7, Canada
}

\begin{abstract}
In this Letter we propose a formation channel for low-mass X-ray binaries with black hole accretors and non-degenerate donors via grazing tidal encounters with subgiants. We estimate that in a typically dense globular cluster with the core density of $10^{5}$ stars $\mathrm{pc}^{-3}$, the formation rates are about one binary per Gyr per 50-100 retained black holes. The donors - stripped subgiants - will be strongly underluminous when compared to subgiant or giant branch stars of the same colors. The products of tidal stripping are underluminous by at least one magnitude for several hundred million years, when compared to normal stars of the same color, and differ from underluminous red stars that could be produced by non-catastrophic mass transfer in an ordinary binary. The dynamically formed binaries become quiescent low-mass X-ray binaries, with lifetimes of about a Gyr. The expected number of $\mathrm{X}$-ray binaries is one per 50-200 retained black holes, while the expected number of strongly underluminous subsubgiant is about half this. The presence of strongly underluminous stars in a globular cluster may be indicative of black holes presence.
\end{abstract}

Keywords: hydrodynamics — stars: black holes — globular clusters: general — binaries: close — X-rays: binaries 


\section{INTRODUCTION}

Radio observations of Milky Way globular clusters (GCs) have indicated that some contain faint potential black-hole (BH) low-mass X-ray binaries (LMXBs). Several BH candidate binaries are under investigation - two LMXBs in M22 (Strader et al. 2012), one in M62 (Chomiuk et al. 2013), one in 47 Tuc (Miller-Jones et al. 2015).

In two cases, white-dwarf (WD) companions are either determined to be plausible (in M22), or are confirmed (in 47 Tuc, Bahramian et al. 2017). This is consistent with an extrapolation that can be made using the observations of very bright extragalactic GC LMXBs, where $L_{x} \gtrsim 10^{39}$ erg $\mathrm{s}^{-1}$ indicates possible $\mathrm{BH}$ accretors. The observational frequency of such very bright extragalactic GC LMXBs is about $(0.7-2) \times 10^{-9}$ per $M_{\odot}$ in massive GCs with an average $M_{\mathrm{V}} \approx-9$ (Kim et al. 2006; Sivakoff et al. 2007; Humphrey \& Buote 2008; Kim et al. 2013). An ultra-compact X-ray $\mathrm{BH}-\mathrm{WD}$ binary, once formed, will remain as a faint $\mathrm{BH}-\mathrm{WD}$ LMXB for $\sim 10^{4}$ times longer than a very bright BH-WD LMXB (Ivanova et al. 2010; Ivanova 2016). This implies that in a dense and massive Milky Way GCs, one faint BH-WD $\mathrm{X}$-ray binary can be expected per $\sim 10^{5} \mathrm{M}_{\odot}$.

However, for faint $\mathrm{BH}-\mathrm{LMXBs}$ with non-degenerate companions, there is no clue regarding their formation frequency from extragalactic observations. There is also a lack of theoretical formation scenarios. A tidal capture of a main sequence star by a BH was previously ruled out, as this should produce a bright source which has not yet been detected (Kalogera et al. 2004). An exchange encounter between a binary and a $\mathrm{BH}$ would make binaries too wide to start mass transfer (MT), if MT starts the duty cycle would be too low to ever be detected (Kalogera et al. 2004).

In this Letter, we propose to examine the formation of a $\mathrm{BH}-\mathrm{LMXB}$ with an underluminous red donor, which is produced by a grazing tidal encounter of a $\mathrm{BH}$ and a low-mass star that recently evolved off its main sequence. The formation of the binary proposed in this manuscript is different from the formation via physical collisions considered in Ivanova et al. (2010) and elsewhere before, where the entire envelope of the subgiant star is lost, and, in the formed binary, the $\mathrm{BH}$ has a degenerate companion.

\section{DYNAMICAL FORMATION}

\subsection{The encounter rate}

$\sigma_{\mathrm{RG}, \mathrm{BH}}$ is the cross-section of an enhanced by gravitational focusing encounter between a red giant $(\mathrm{RG})$ and a $\mathrm{BH}$ (here we include subgiants):

$$
\begin{aligned}
\sigma_{\mathrm{RG}, \mathrm{BH}} & =\pi r_{\mathrm{enc}}^{2}\left(1+\frac{2 G\left(M_{\mathrm{RG}}+M_{\mathrm{BH}}\right)}{r_{\mathrm{enc}} v_{\infty}^{2}}\right) \\
& \approx \pi r_{\mathrm{enc}}^{2}\left(1+3800 \frac{\left(M_{\mathrm{RG}}+M_{\mathrm{BH}}\right) / M_{\odot}}{r_{\mathrm{enc}} / R_{\odot}\left(v_{\infty} / 10 \mathrm{~km} / \mathrm{s}\right)^{2}}\right)
\end{aligned}
$$

Here $r_{\text {enc }}$ is the largest closest approach that would lead to a specific strong encounter, and $v_{\infty}$ is the relative velocity at infinity.

We search for an encounter such that at periastron the stars pass relatively close, and it is possible to damp enough of the initial kinetic energy into the non-degenerate star to form a bound binary, $r_{\text {enc }} \leq r_{\text {form}}$. On the other hand, an encounter should be not too close, so stars neither collide, nor the envelope is removed completely during the encounter, where only a naked core that would become later a WD is left, $r_{\mathrm{enc}} \geq r_{\mathrm{wd}}$.

For the range of closest approaches $r_{\mathrm{wd}}<r_{\mathrm{enc}} \leq r_{\text {form }}$ (we will determine the values in $\S 2.2$ ), and assuming that $r_{\text {form }}$ is of order of several solar radii, while $v_{\infty}$ in GCs is of order of $10 \mathrm{~km} / \mathrm{s}$, we obtain:

$\sigma_{\mathrm{RG}, \mathrm{BH}}^{\text {form }} \approx 1.2 \times 10^{4} R_{\odot}{ }^{2} \frac{M_{\mathrm{RG}}+M_{\mathrm{BH}}}{M_{\odot}}\left(\frac{10 \mathrm{~km} / \mathrm{s}}{v_{\infty}}\right)^{2} \frac{r_{\mathrm{form}}-r_{\mathrm{wd}}}{R_{\odot}}$.

The total number of encounters leading to this kind of binary formation, per $\mathrm{BH}$, and per unit of time:

$$
N^{\text {form }}=n_{\mathrm{RG}} \sigma_{\mathrm{RG}, \mathrm{BH}}^{\text {form }} v_{\infty},
$$

where $n_{\mathrm{RG}}=f_{\mathrm{RG}} n_{\mathrm{c}}$ is the number density of RGs, $f_{\mathrm{RG}}$ is the fraction of RGs in the stellar population on a GC core, typically 8-10 percent (Ivanova et al. 2005), and $n_{\mathrm{c}}$ is the number density in the core of a GC. Scaling to a typical massive GC where core's number density is $10^{5}$ per $p c^{3}$, and using $n_{5}=n_{\mathrm{c}} / 10^{5}$, we have:

$N^{\mathrm{form}}=6 \times 10^{-3} f_{\mathrm{RG}} n_{5} \frac{M_{\mathrm{RG}}+M_{\mathrm{BH}}}{M_{\odot}} \frac{10 \mathrm{~km} / \mathrm{s}}{v_{\infty}} \frac{r_{\text {form }}-r_{\mathrm{wd}}}{R_{\odot}} \mathrm{Gyr}^{-1}$,

A preliminary estimate of at which distance this capture could take place can be done using fitting functions for the tidal energy dissipated during a stellar encounter, $E_{\text {tide }}\left(r_{\mathrm{p}}\right)$ (Portegies Zwart \& Meinen 1993). Here $r_{\mathrm{p}}$ is the closest initial approach. To form a bound binary, the initial relative kinetic energy of the two stars $E_{\text {kin }}$ has to be damped. This defines the maximum closest approach $r_{\text {form }}$ at which the encounter leads to a binary formation, $E_{\text {tide }}\left(r_{\text {form }}\right)>E_{\text {kin }}$. As we are interested only in cases when a part of the envelope is left, the amount of energy that must dissipate should not exceed (by absolute value) RG envelope's binding energy $E_{\text {env }}$. This defines the minimum closest approach $r_{\mathrm{wd}}$ at which the encounter does not fully eject the envelope, $E_{\text {tide }}\left(r_{\text {wd }}\right)+E_{\text {env }}<0$.

For a preliminary estimate, we use analytic formulae for stellar evolution (Hurley et al. 2000), and adopt that $E_{\mathrm{env}}=$ $-G M_{\mathrm{RG}, \text { env }} M_{\mathrm{RG}} / r_{\mathrm{RG}}$, where $M_{\mathrm{RG}, \text { env }}$ is the $\mathrm{RG}$ envelope mass, and $r_{\mathrm{RG}}$ is the radius of the RG. At $10 \mathrm{Gyr}$, the RG population is formed by stars of nearly identical initial mass. 
The probability of the presence (at this moment of time) of giants that have radii within a specific range is hence about the same as the ratio of how long the giant could exist in that specific radii range, to its total lifetime as a giant. The timeaveraged range of $r_{\mathrm{enc}}$ that leads to the binary formation can be found by calculating $r_{\text {form }}$ and $r_{\text {wd }}$ for each giant's evolutionary moment, and integrating over time. For a BH mass of $7 M_{\odot}$ and $v_{\infty}=10 \mathrm{~km} / \mathrm{s}$ (we will use these values as default everywhere thereafter), $\overline{r_{\text {form }}-r_{\mathrm{wd}}} \approx 8 R_{\odot}$. If we increase the mass of the $\mathrm{BH}$, this value slowly increases, but does not exceed $\sim 9.6 R_{\odot}$ for BHs less massive than $100 M_{\odot}$. This estimate implies that, with a typical RG fraction of about $10 \%$, typical RG mass of $1 M_{\odot}$, and core number density of $10^{5}$ per $p c^{3}$, one stripped RG can be formed per 25 within-the-corelocated BHs per Gyr.

We find that $80 \%$ of the encounters that form a binary take place while the RG is smaller than $10 R_{\odot}$, and $50 \%$ of all encounters are taking place when the RG is smaller than $4 R_{\odot}$ - the role of the well evolved giants is small, and subgiants are the most important. As an example, for a $\mathrm{RG}$ of $1 M_{\odot}$ that has $r_{\mathrm{RG}} \sim 2 R_{\odot}, r_{\text {form }}-r_{\mathrm{wd}} \approx 5 R_{\odot}$ (where $\left.r_{\mathrm{wd}} \sim 3 R_{\odot}\right)$. However, how much energy is used to directly remove part of the envelope, and how much is simply absorbed by the envelope to make it less bound, and hence the actual values of $r_{\text {form }}-r_{\mathrm{wd}}$, can only be found by detailed 3D simulations.

\subsection{Three-dimensional hydrodynamical simulations}

Here we consider encounters of a $7 M_{\odot}$ BH with a RG that has the mass of $1 M_{\odot}$, the radius of $2 R_{\odot}$, and the core of $\sim 0.13 M_{\odot}$. To model encounters, we follow the framework described in details in Nandez et al. (2014). To evolve the RG and find the initial one-dimensional (1D) stellar profile, we use the TWIN/Star stellar code (recent updates described in Glebbeek et al. 2008). For three-dimensional (3D) simulations, we use the smoothed particle hydrodynamics (SPH) code STARSMASHER (Gaburov et al. 2010; Lombardi et al. 2011). STARSMASHER has been modified in the past to accept TWIN/Star stellar models (Nandez et al. 2014). We model the 3D star using 50K regular SPH particles and one special particle that represents the core; the core mass is $0.13 M_{\odot}$. This special particle only interacts gravitationally with other particles.

To set up the collision of this star and a $7 M_{\odot} \mathrm{BH}$, the two stars are given relative velocity at infinity of $10 \mathrm{~km} / \mathrm{s}$, and their locations are such that the resulting periastron distances are 2.5,3.0,3.25, 3.5, 3.75, 4.0 and $4.25 R_{\odot}$. As we model the entire orbit of the formed binaries, the simulations are very computationally expensive when compared to only modeling the periastron passages. We may expect that increase of the resolution by a factor of two could provide a clarification of the post-encounter binary semi-major axis by a few \% (see, e.g., Nandez et al. 2014).

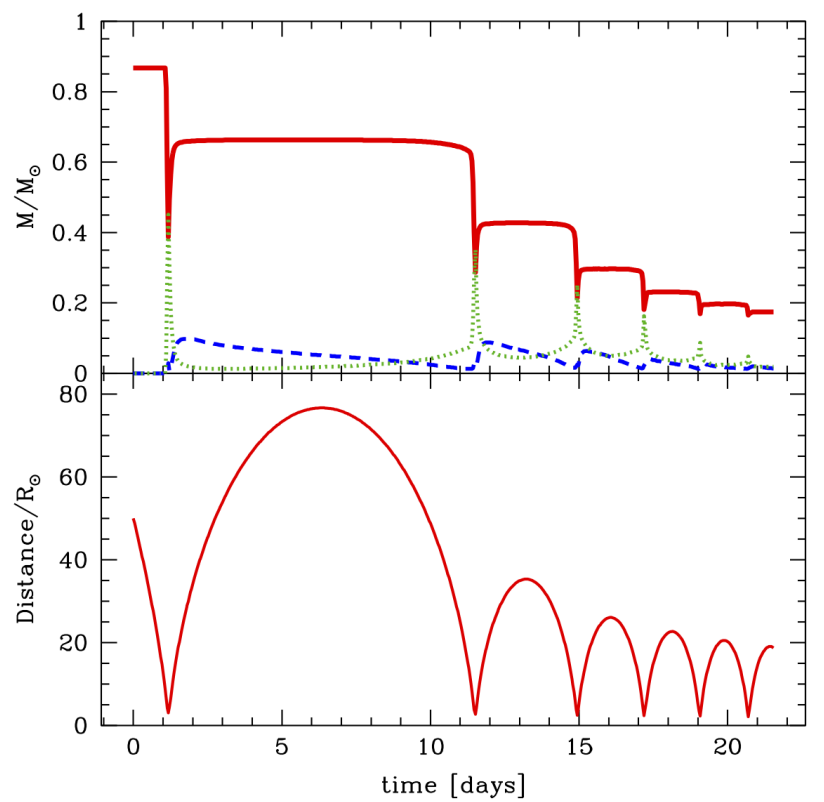

Figure 1. The lower panel shows the instantaneous distance between the RG core and the BH. The upper panel shows the envelope mass that is within the instantaneous RL located around the RG core including other envelope particles bound to that RL (red solid line), the total mass within the RL of the BH excluding the BH itself (blue dashed line), and the mass that is still bound to the binary but is located outside of either of the instantaneous RLs (green dotted line).

A typical simulation is shown in Figure 1, using as an example the case with $r_{p}=3 R_{\odot}$. The two stars, after the initial passage, formed a bound binary system. The semi-major axis in this binary is continuing to decrease. During the sixth periastron passage, the decrease of the distance between the $\mathrm{RG}$ core and the $\mathrm{BH}$ is already only a few percent if compared to the previous passage. This is because almost all remaining envelope mass is contained within the instantaneous Roche lobe (RL) that the RG has at the periastron. We define the instantaneous RL of the RG (or of the $\mathrm{BH}$ ) as a $3 \mathrm{D}$ equipotential surface that is formed by the RG core itself (or by the $\mathrm{BH}$ ) and all the particles that are bound to the RG core (or the $\mathrm{BH}$ ), adopting that the two objects are within an "instantaneous" corotating frame that is defined by the separation between the RG center of mass and the BH. As the cumulative mass of SPH particles bound to the RG core is non-negligible and affects the size of the RL, the exact 3D shape of each RL is found iteratively. The envelope mass decrease during the sixth passage is $0.019 M_{\odot}$. Some particles are becoming bound to the $\mathrm{BH}$, but most of them are ejected from the system as the two stars move towards their apastron. By the end of the sixth passage, only about $0.01 M_{\odot}$ remains bound to the $\mathrm{BH}$.

The formed binary has a semi-major axis of $10.5 R_{\odot}$ and an eccentricity of $e=0.8$; the retained envelope mass is 
$0.174 M_{\odot}$. The minimum mass that the $\mathrm{RG}$ envelope has during the last two periastron passages is not changing and is $0.17 M_{\odot}$. All that mass is located within $93 \%$ of the instantaneous RL that is made by the RG core and the other particles bound to RG core during the periastron passage. The distance to the $\mathrm{BH}$ during the periastron passage is about 4.7 times larger than the stripped RG. The particles of the envelope therefore are not affect much by tidal perturbations from the $\mathrm{BH}$ during the periastron passage. The binary may be considered formed with the stripped giant having a mass of $0.3 M_{\odot}$. We note that at the current the radius of the stripped giant is about $0.3 R_{\odot}$, while the same mass was contained within about $0.22 R_{\odot}$ at the start of $3 \mathrm{D}$ simulations. We find that the envelope (that remains bound) has its entropy almost unchanged, as compared to the unperturbed star, for the same mass coordinate.

The model with $r_{p}=4.25 R_{\odot}$ did not result in a binary formation despite the encounter stripping $0.09 M_{\odot}$. The model with $r_{p}=4 R_{\odot}$ resulted in a formation of a close binary, with $\sim 0.11 M_{\odot}$ ejected during the first periastron passage, and an initial semi-major axis of about $110 R_{\odot}$. The semi-major axis will decrease by a few times, as shown in the model with $r_{p}=3 R_{\odot}$ (Fig. 1). Based on the studies with smaller $r_{p}$, we estimate that the final minimum amount of the stripped mass is expected to be at least $\sim 0.32 M_{\odot}$, as this is the mass that was was outside of the giant's instantaneous RL yet during the first periastron approach. As the inner layers expand in all the simulations by $\sim 30 \%$ as compared to their initial radius coordinate, the stripped mass is expected to be $\gtrsim 0.4 M_{\odot}$. It implies that one can expect a creation of stripped giants with a non-continuous amount of stripped mass: less than $0.1 M_{\odot}$ is stripped when a binary is not formed, and at least $0.4 M_{\odot}$ is stripped when a binary is formed.

On the other hand, our simulation with the smallest approach at periastron did not result in a complete envelope removal even after six periastron passages, where the mass lost during the sixth passage is only $0.008 M_{\odot}$, and the semimajor axis decreased by only $1.6 \%$. From this it follows that in our analytic estimate for $r_{\mathrm{wd}}$, we either underestimated the energy required for the envelope removal, or overestimated the energy that can be placed into the envelope during a tidal perturbation. If at the periastron the $\mathrm{BH}$ is too far away to impose a tidal perturbation on the envelope's layers located close to the core, this part of the envelope is not removed during 3D simulations. The final binary has a semi-major axis of $7.4 R_{\odot}$, an eccentricity of $e=0.77$, and the envelope mass is $0.146 M_{\odot}$. While we do not fine-tune the exact value of $r_{\mathrm{wd}}$ further, it is apparent that the rate of formation of stripped RGs is lower than we estimated in $\S 2.1$ by a factor of few: from 3D, we have $r_{\text {form }}-r_{\mathrm{wd}} \gtrsim 1.5 R_{\odot}$, while the simple estimate have predicted $r_{\text {form }}-r_{\mathrm{wd}} \approx 5 R_{\odot}$; this reduces the total formation rate by a similar factor of 2 to 4 .

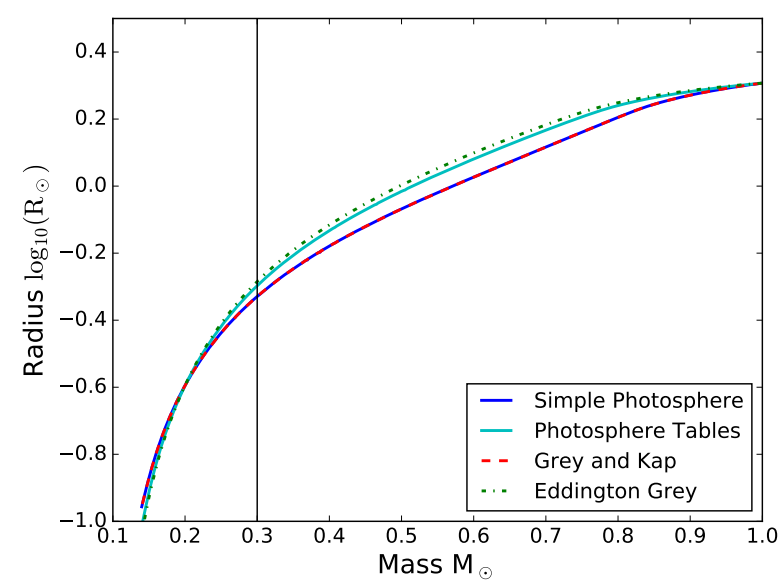

Figure 2. The radii of a $1 M_{\odot}$ and $2 R_{\odot}$ subgiant while it is evolved with mass loss of $1 M_{\odot} \mathrm{yr}^{-1}$, for four atmospheric boundary conditions. "Simple photosphere" stands for an estimate at the optical depth $\tau=2 / 3$; in the second choice the precalculated atmosphere tables for photospheres are used; "Grey and kap" means that a simple grey atmosphere is calculated to to find consistent pressure $P$, temperature $T$, and opacity at the surface; and "Eddington Grey" uses Eddington $T-\tau$ integration

(see MESA instrument papers for more details on the used ABCs).

\section{PROPERTIES OF THE STRIPPED STAR AFTER AN ENCOUNTER}

To create and evolve stripped remnants, we used the 1D stellar evolution code MESA (Modules for Experiments in Stellar Astrophysics, see instrument papers Paxton et al. 2011, 2013, 2015), revision 8677. While the structure of the low-mass subgiants obtained with MESA is very similar to the one obtained with TWIN/Star, only with MESA are we able to strip the subgiant at a mass loss rate comparable to dynamical stripping during the encounter.

We evolved a star with the mass of $1 M_{\odot}$ and metallicity of $Z=0.01$. The evolutionary calculations are done using the default assumptions as in MESA. When the star expanded to the radius of $2 R_{\odot}$, we enforced rapid mass loss of the envelope, at the rate of $1 M_{\odot}$ per year.

We found that the radius of the stripped star during the mass loss, for each given instantaneous total mass, depends on the atmospheric boundary conditions (ABCs) that are used, see Figure 2. There, we used four ABCs, selected from the large set of conditions provided with $\mathrm{MESA}$, making sure that all of the used ABCs are theoretically valid for studies of low-mass stars, and thoroughly tested in previous studies of low-mass stars by various groups.

Independently from the ABC, we cannot obtain as small a radius in our 1D stripped product as was obtained during an encounter using the $3 \mathrm{D}$ code. The smallest radius that we have obtained with $\mathrm{MESA}$-stripping for a $0.3 M_{\odot}$ remnant is $0.47 R_{\odot}$, while it is $0.32 R_{\odot}$ with the $3 \mathrm{D}$ code. We link 

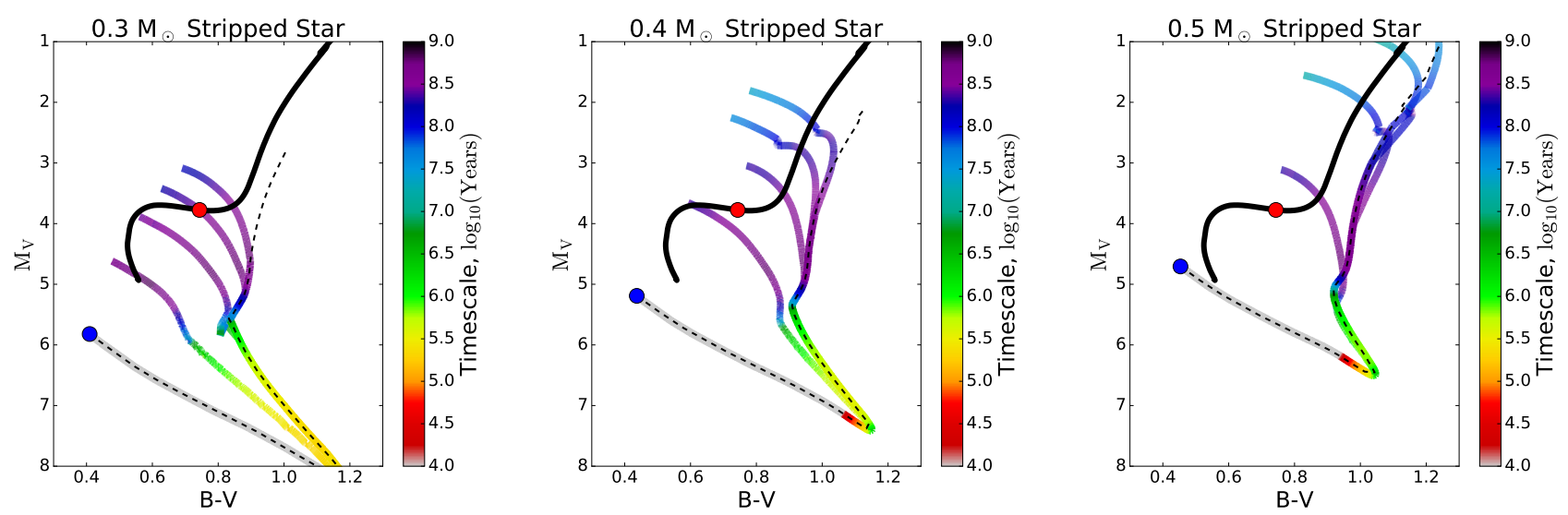

Figure 3. The evolution of the stripped subgiant. The position of the $1 M_{\odot}$ and $2 R_{\odot}$ subgiant prior the stripping is indicated with the red circle. The location of the stripped star right after the stripping is indicated with the blue circle. The black solid line shows the evolutionary track of a $1 M_{\odot}$ star. The black dashed line shows the evolution of the stripped star as it a single star. Tracks with the colors show the evolution during the MT, shown only until the remnant has detached. The colors of the tracks indicate instantaneous characteristic timescales, such that it take to change either the magnitude $M_{V}$ by one, or color $B-V$ by 0.2 . The left panel shows the case of stripping down to $0.3 M_{\odot}$ (the initial orbital periods are $P_{\text {orb }}=0.21,0.54,0.97$ and 1.46 days, from the left to the right), the middle panel shows the case of stripping down to $0.4 M_{\odot}$ $\left(P_{\text {orb }}=0.32,0.88,1.62\right.$ and 2.48 days $)$, and the right panel shows the case of stripping down to $0.5 M_{\odot}\left(P_{\text {orb }}=0.42,1.98,4.22\right.$ and 6.95 days $)$.

this different behavior to the entropy of the very outer layers, with the mass $\leq 10^{-4} M_{\odot}$. The 1D star inside that very outer layer has its entropy unchanged, like the product of the 3D encounter. The entropy of this very outer layer in a 1D star has increased compared to its initial value, by 1 to 45 percent, resulting in star's expansion, while in 3D, the generation of this entropy is not observed. On the one hand, this tiny surface layer might be capable of losing energy radiatively on a timescale of one year, the timescale for the mass loss we used in MESA. Hence, it might be expected that the presence of a rarefied but expanded shell of a tiny mass is physically motivated. On the other hand, the timescale of the mass loss in $3 \mathrm{D}$ is about 10 times faster than in MESA, where a purely dynamical mass loss was not possible, and it might be that the observed thermal adjustment would not happen in nature. In any case, dynamically, a layer of this mass cannot affect the shrinkage of the binary.

The binary that is formed in our 3D simulations is highly eccentric. During the binary periastron approach, the stripped star is at its RL overflow. We note that the mass of the stripped star is too small to warrant the transfer of a substantial part of the orbital angular momentum $J_{\text {orb }}$ into the remaining envelope, and hence $J_{\text {orb }}$ will remain mainly in the binary orbit. Therefore, if the binary would circularize, its semi-major axis will be close to $a_{\text {per }}(1+e)$, where $e$ is the post-encounter eccentricity. In principle, it was shown that for the mass ratio as in our system, both eccentricity and semi-major axis may grow in case of impulsive RL overflow at the periastron (Sepinsky et al. 2009). However, we note that a self-consistent treatment of the MT in eccentric binaries is not yet implemented in MESA or other stellar binary codes.
We choose to consider the limiting cases, and to see how different could be the appearance of the stripped star within those limits. First, we consider how the stripped star evolves by itself - in this case, the remnant will be maximally "relaxed" while it evolves after the encounter. Second, we consider the case of a non-eccentric binary where the MT starts immediately after the mass stripping (the stripped star radius is the same as its RL). In this case $a=a_{\min }=a_{\text {per }}$ and the remnant will be evolving as maximally "perturbed" (this is not a likely case of the post-encounter evolution due to angular momentum conservation). Third, we consider the case of a binary that has semi-major axis the same as is found from 3D simulations. This semi-major axis is likely larger than it would be in Nature. And we consider intermediate cases, with the semi-major axis chosen between the minimum as above, and the maximum that is found from 3D simulations. In all the cases, we adopt that the systems are non-eccentric, and hence are evolving to the start of the MT via RL overflow mainly via remnant's expansion and magnetic braking. For magnetic braking, we use default MESA prescription for magnetic braking from Rappaport et al. (1983) with $\gamma=4$. We adopted that the MT in our binaries is non-conservative, where the mass accretion rate is limited by the Eddington limit, and the excess mass is lost from the system with the specific angular momentum of the accretor.

The tracks for the $0.3 M_{\odot}$ stripped star are shown in Figure 3. Note that the two limiting cases, maximally relaxed and maximally perturbed, restrict the parameter space within which the stripped star can appear on a color magnitude diagram (CMD), and the most likely tracks are in between the two limiting cases. During the MT, the star evolves from the luminosities that are lower than those of the subgiant branch 
into higher luminosities and hotter temperatures. We define as underluminosity $\delta M_{\mathrm{V}}^{\text {und }}=M_{\mathrm{V}}^{\text {strip }}-M_{\mathrm{V}}^{\text {norm }}$ as the difference between the magnitude of the stripped star, $M_{\mathrm{V}}^{\text {strip }}$, and an unperturbed star of a similar color, $M_{\mathrm{V}}^{\text {norm }}$. In our case, normal subgiants have $M_{\mathrm{V}}^{\text {norm }} \approx 3.8$ at $B-V=0.8$. The stripped product has $\delta M_{\mathrm{v}}^{\text {und }} \gtrsim 2$ for a few dozen million years after the stripping, while $1 \lesssim M_{\mathrm{v}}^{\text {und }} \lesssim 2$ for $\sim 3 \times 10^{8}$ years.

In encounters with stripping down to $0.4 M_{\odot}$ and $0.5 M_{\odot}$, the formed binaries have slightly larger semi-major axis than in the case of the star stripped down to $0.3 M_{\odot}$. Similar to the case of $0.3 M_{\odot}$ stripped star, $0.4 M_{\odot}$ stripped star have $\delta M_{\mathrm{v}}^{\text {und }} \gtrsim 1$ for $\sim 1.5 \times 10^{8}$ years, but $0.5 M_{\odot}$ stripped star is strongly underluminous only for less than $10^{7}$, if compared to subgiants. However, $0.5 M_{\odot}$ remnants are also redder. They are underluminous by $\delta M_{\mathrm{v}}^{\text {und }} \gtrsim 2$ for $\sim 8 \times 10^{8}$ years, if compared to giants that have similar colors (see Figure 3 ).

We verified that a similarly long-living strongly underluminous star can be made by stripping of $60 \%$ or more of the donor's mass at a time-averaged $\dot{M} \gtrsim 10^{-6} M_{\odot} \mathrm{yr}^{-1}$. A time-averaged $\dot{M}>10^{-6} M_{\odot} \mathrm{yr}^{-1}$ from a low-mass subgiant or giant can be supported for the duration of the stripping more than a half of the primary mass only if the donor remains more massive than the companion for most of the mass transfer episode. A mass transfer with such initial mass ratio is expected to be dynamical unstable.

During the MT, an X-ray binary can appear either as a persistent, or as a transient source. In the first case the X-ray luminosity is expected to be roughly proportional to the mass accretion rate, while a transient source spends most of the time in quiescence, and has very low X-ray luminosity, typically of the order of $10^{33} \mathrm{ergs} / \mathrm{s}$. The bifurcation between appearing as a persistent or a transient source depends on how stable is the accretion disk (Menou et al. 2000; Dubus et al. 2001). Using the revised disk instability model (Coriat et al. 2012), we find that for most of the time our MT binaries will be in the unstable regime, and hence will appear as quiescent LMXBs (qLMXBs).

The contribution of the accretion disk to the optical luminosity during quiescence can be estimated using observations of V404 Cyg, the low-mass X-ray binary with an orbital period of 6.47 days and a $\mathrm{BH}$ companion of $9 \pm_{0.6}^{0.2} M_{\odot}$ (Bernardini et al. 2016). In V404 Cyg, the maximum contribution of the disk in any optical band was estimated to not exceed $14 \%$ of the flux in that band (Shahbaz et al. 1996). As the most likely value of V404 Cyg's secondary's absolute magnitude is $M_{V}=3.4$ (Shahbaz et al. 1994), the upper limit on the disk luminosity is thus $M_{V} \approx 5.5$, and hence the accretion disk potentially can contribute to the total optical luminosity during the first few million years after the encounter.

\section{CONCLUSIONS}

We have considered the formation channel of LMXBs in GCs through grazing encounters between BHs and RGs. We used a multi-step approach where we first evaluated the total cross-section of encounters that can lead to the formation of binaries with a stripped giant companion using the analytical formulae for the tidal energy dissipation and for stellar evolution. Second, we clarified the predictions for the range of the closest approaches by performing 3D hydrodynamical simulations that used the structure of a $2 R_{\odot}$ giant with a mass of $1 M_{\odot}$ as provided by a fully-fledged $1 \mathrm{D}$ stellar evolutionary code. To find how the product would look like observationally, we evolved the stripped product in a binary using a 1D stellar evolutionary code.

The formation rate is about one binary per Gyr per 50100 remaining $\mathrm{BHs}$ (if the core number density is $\sim 10^{5}$ $p c^{-3}$ ). Theoretical predictions vary on how many BHs could be present: from a couple of dozens of BHs in a GC like M22 (Sippel \& Hurley 2013), to several hundreds of BHs in a cluster that would have a central density about $10^{5}$ stars per $\mathrm{pc}^{3}$, and a mass of a few times $10^{5} M_{\odot}$ (e.g., see all models starting with "n8" from Morscher et al. 2015). Our formation channel will be efficiently productive within this predicted range for the retained $\mathrm{BHs}$.

The formed binary unavoidably starts the MT from the captured and stripped subgiant, while appearing most of the time as a qLMXB. The donor in such qLMXB is strongly underluminous, by $\delta M_{\mathrm{V}}^{\mathrm{und}} \gtrsim 1$, for a few hundred million years. Then after a few hundred million years, our stripped stars will evolve to larger luminosities and will blend with other exotic stars of a GC, e.g. subsubgiants or red stragglers formed via various channels considered in Leiner et al. (2017). The total timescale of the MT is about $0.5-1$ Gyr. The expected number of present qLMXBs is one per $50-200 \mathrm{BHs}$ retained in a GC core.

We find that the amount of stripped mass in the encounters which did not result in a binary formation, and in those that have resulted in a binary formation, is very different, by at least $0.3 M_{\odot}$. Neutron stars may also be capable of acquiring companions in a similar manner, while other stars would merge with the subgiant during an encounter that would be close enough for the tidal damping to work, and for the substantial part of the envelope to be ejected. A timeaveraged mass loss rate that can produce as extreme longliving outliers (for the same colors) as our stripped stars is $\gtrsim 10^{-6} M_{\odot} \mathrm{yr}^{-1}$, and it has to remove $\gtrsim 60 \%$ of the donor mass; such mass transfer would not proceed stably in ordinary binaries of that age in the GC. Therefore, the presence of a red star that is strongly underluminous, $M_{\mathrm{V}}^{\text {und }}>1$, may indicate that it has a $\mathrm{BH}$ or a NS companion. If a GC qLMXB in a $\mathrm{GC}$ is implied to have a $\mathrm{BH}$ accretor, by the ratio of its radio luminosity to its $\mathrm{X}$-ray luminosity, we propose that it is likely to have a donor formed by the considered channel. 
In about a half of the cases, the donor is expected to remain strongly underluminous, and this is how its counterpart might be identified.

NI acknowledges support from CRC program, and funding from NSERC Discovery. NI and KV acknowledge that a part of this work was performed at the KITP which is sup- ported in part by the NSF under Grant No. NSF PHY1125915. CAR acknowledges for support Science without Borders. JLAN acknowledges CONACyT for its support. The authors thank the anonymous referees for the comments that improved the clarity of the manuscript. This research has been enabled by the use of computing resources provided by Compute/Calcul Canada and the Shared Hierarchical Academic Research Computing Network (SHARCNET).

\section{REFERENCES}

Bahramian, A., Heinke, C. O., Tudor, V., et al. 2017, MNRAS, 467, 2199

Bernardini, F., Russell, D. M., Shaw, A. W., et al. 2016, ApJL, 818, L5

Chomiuk, L., Strader, J., Maccarone, T. J., et al. 2013, ApJ, 777, 69

Coriat, M., Fender, R. P., \& Dubus, G. 2012, MNRAS, 424, 1991

Dubus, G., Hameury, J.-M., \& Lasota, J.-P. 2001, A\&A, 373, 251

Gaburov, E., Lombardi, Jr., J. C., \& Portegies Zwart, S. 2010, MNRAS, 402, 105

Glebbeek, E., Pols, O. R., \& Hurley, J. R. 2008, A\&A, 488, 1007

Humphrey, P. J., \& Buote, D. A. 2008, ApJ, 689, 983

Hurley, J. R., Pols, O. R., \& Tout, C. A. 2000, MNRAS, 315, 543

Ivanova, N. 2016, Star Clusters and Black Holes in Galaxies across Cosmic Time, 312, 203

Ivanova, N., Chaichenets, S., Fregeau, J., et al. 2010, ApJ, 717, 948

Ivanova, N., Rasio, F. A., Lombardi, Jr., J. C., Dooley, K. L., \&

Proulx, Z. F. 2005, ApJL, 621, L109

Kalogera, V., King, A. R., \& Rasio, F. A. 2004, ApJL, 601, L171

Kim, D.-W., Fabbiano, G., Ivanova, N., et al. 2013, ApJ, 764, 98

Kim, E., Kim, D.-W., Fabbiano, G., et al. 2006, ApJ, 647, 276

Leiner, E., Mathieu, R. D., \& Geller, A. M. 2017, ArXiv e-prints, arXiv: 1703.10181

Lombardi, Jr., J. C., Holtzman, W., Dooley, K. L., et al. 2011, ApJ, 737, 49

Menou, K., Hameury, J.-M., Lasota, J.-P., \& Narayan, R. 2000, MNRAS, 314, 498
Miller-Jones, J. C. A., Strader, J., Heinke, C. O., et al. 2015, MNRAS, 453, 3918

Morscher, M., Pattabiraman, B., Rodriguez, C., Rasio, F. A., \& Umbreit, S. 2015, ApJ, 800, 9

Nandez, J. L. A., Ivanova, N., \& Lombardi, Jr., J. C. 2014, ApJ, 786,39

Paxton, B., Bildsten, L., Dotter, A., et al. 2011, ApJS, 192, 3

Paxton, B., Cantiello, M., Arras, P., et al. 2013, ApJS, 208, 4

Paxton, B., Marchant, P., Schwab, J., et al. 2015, ApJS, 220, 15

Portegies Zwart, S. F., \& Meinen, A. T. 1993, A\&A, 280, 174

Rappaport, S., Verbunt, F., \& Joss, P. C. 1983, ApJ, 275, 713

Sepinsky, J. F., Willems, B., Kalogera, V., \& Rasio, F. A. 2009, ApJ, 702, 1387

Sippel, A. C., \& Hurley, J. R. 2013, MNRAS, 430, L30

Sivakoff, G. R., Jordán, A., Sarazin, C. L., et al. 2007, ApJ, 660, 1246

Shahbaz, T., Ringwald, F. A., Bunn, J. C., et al. 1994, MNRAS, 271, L10

Shahbaz, T., Bandyopadhyay, R., Charles, P. A., \& Naylor, T. 1996, MNRAS, 282, 977

Strader, J., Chomiuk, L., Maccarone, T. J., Miller-Jones, J. C. A., $\&$ Seth, A. C. 2012, Nature, 490, 71 